\title{
Cryopreservation of in vitro matured oocytes after ex vivo oocyte retrieval from gynecologic cancer patients undergoing radical surgery
}

\author{
Chan Woo Park', Sun Hee Lee ${ }^{2}$, Kwang Moon Yang ${ }^{1}$, In Ho Lee ${ }^{3}$, Kyung Teak Lim³ ${ }^{3}$ Ki Heon Lee ${ }^{3}$, Tae Jin Kim³ \\ 'Division of Reproductive Endocrinology and Infertility, ${ }^{2}$ Laboratory of Reproductive Biology and Infertility, ${ }^{3}$ Division of Gynecologic Oncology, \\ Department of Obstetrics and Gynecology, Cheil General Hospital and Women's Healthcare Center, Dankook University College of Medicine, Seoul, \\ Korea
}

Objective: The aim of this study was to report a case series of in vitro matured (IVM) oocyte freezing in gynecologic cancer patients undergoing radical surgery under time constraints as an option for fertility preservation (FP).

Methods: Case series report. University-based in vitro fertilization center. Six gynecologic cancer patients who were scheduled to undergo radical surgery the next day were referred for FP. The patients had endometrial $(n=2)$, ovarian $(n=3)$, and double primary endometrial and ovarian $(n=1)$ cancer. Ex vivo retrieval of immature oocytes from macroscopically normal ovarian tissue was followed by mature oocyte freezing after IVM or embryo freezing with intracytoplasmic sperm injection.

Results: A total of 53 oocytes were retrieved from five patients, with a mean of 10.6 oocytes per patient. After IVM, a total of 36 mature oocytes were obtained, demonstrating a $67.9 \%$ maturation rate. With regard to the ovarian cancer patients, seven IVM oocytes were frozen from patient 3, who had stage IC cancer, whereas one IVM oocyte was frozen from patient 4, who had stage IV cancer despite being of a similar age. With regard to the endometrial cancer patients, 15 IVM oocytes from patient 1 were frozen. Five embryos were frozen after the fertilization of IVM oocytes from patient 6.

Conclusion: Immature oocytes can be successfully retrieved ex vivo from macroscopically normal ovarian tissue before radical surgery. IVM oocyte freezing provides a possible FP option in patients with advanced-stage endometrial or ovarian cancer without the risk of cancer cell spillage or time delays.

Keywords: Cryopreservation; In vitro oocyte maturation techniques; Ovarian neoplasm; Uterine neoplasm

\section{Introduction}

Uterine and ovarian cancer comprise a significant proportion of malignancies affecting women [1]. Despite predominantly occurring in postmenopausal women, approximately $20 \%$ of gynecologic ma-

Received: Feb 25, 2016 · Revised: Mar 15, 2016 · Accepted: Apr 14, 2016 Corresponding author: Tae Jin Kim

Division of Gynecologic Oncology, Department of Obstetrics and Gynecology, Cheil General Hospital and Women's Healthcare Center, Dankook University College of Medicine, 17 Seoae-ro 1-gil, Jung-gu, Seoul 04619, Korea Tel: +82-2-2000-7577 Fax:82-2-2000-7183 E-mail:kimonc@hotmail.com

This is an Open Access article distributed under the terms of the Creative Commons Attribution Non-Commercial License (http://creativecommons.org/licenses/by-nc/4.0/) which permits unrestricted non-commercial use, distribution, and reproduction in any medium, provided the original work is properly cited. lignancies take place in women of reproductive age who have yet to complete family planning [2-5]. When broken down by subtype, $8 \%$ of endometrial cancers, $12 \%$ of ovarian cancers, and $40 \%$ of cervical cancers occur in patients during their childbearing years [6]. Improvements in cancer diagnosis and treatment have resulted in an improved survival rate, allowing these patients to focus on their quality of life. Fertility preservation (FP) has become an important issue for cancer patients of reproductive age, and planning for the possibility of parenthood after cancer treatment and FP is now an integral component of pre-cancer treatment counseling.

In gynecologic malignancies, fertility-sparing surgery is a viable option for certain early-stage cancer patients, including those with early cervical carcinoma, early endometrial adenocarcinoma, ovarian tu- 
mors of low malignancy, and some selected cases of unilateral ovarian carcinoma (stage IA) [3,7]. A conservative approach to fertility involves sparing the uterus and a unilateral ovary during surgery. However, in cases of advanced gynecologic malignancies, reproductive organs such as the uterus and ovaries must be removed by radical surgery. FP for gynecologic cancer patients undergoing radical surgery poses a significant clinical challenge. Radical surgery is often initiated without considering FP because neither cancer patients nor their oncologists wish to delay surgical treatment. Furthermore, the removal of reproductive organs through radical surgery makes it difficult to consider future pregnancies. When advanced cancer patients are faced with FP, debates can arise over which FP option is preferable or whether cancer cell spillage could be prevented during FP procedure.

In cases of in situ ovarian cancer or endometrial cancer with the potential for ovarian involvement, the only option for FP is obtaining oocytes for either oocyte or embryo freezing. In vitro matured (IVM) oocyte freezing is an option in the case of time constraints, such as if an operation is scheduled for the next day [8]. Immature oocytes can be retrieved in vivo or ex vivo, matured in vitro, and frozen by vitrification. Ex vivo oocyte collection is preferred to in vivo collection in order to avoid the possibility of cancer cell spillage. A few reports have discussed ex vivo retrieval in advanced-stage gynecologic cancer patients [9-11]. Prasath et al. [11] reported the first pregnancy and live birth resulting from cryopreserved embryos obtained from IVM oocytes after oophorectomy in an ovarian cancer patient.

Herein, we report a case series of FP using IVM oocytes retrieved from macroscopically normal ovarian tissue harvested during radical surgery.

\section{Methods}

The subjects of this study included six gynecologic cancer patients who were referred by their gynecologic oncologists for FP consultation. The patients had endometrial $(n=2)$, ovarian $(n=3)$, and double primary endometrial and ovarian $(n=1)$ cancer. At the time of consultation, all patients were planning to undergo radical surgery the next day; therefore, all the patients had a time constraint of less than 24 hours. For FP, we planned oocyte freezing for unmarried patients and embryo freezing for a married patient. After discussions with the gynecologic oncologist, each patient planned to have oocytes harvested from macroscopically normal ovarian tissue during radical surgery, followed by IVM.

All subjects provided written informed consent and parental consent was also obtained in the case of one patient who was not married. The gynecologic oncologist explained the experimental nature of this procedure and all patients were clearly informed that a surro- gate mother would be required for future conception after cancer treatment.

For the endometrial cancer patients, total hysterectomy (TH), bilateral salpingo-oophorectomy (BSO), bilateral pelvic lymph node dissection (BPLND), and bilateral para-aortic lymph node dissection (BPALND) were performed. Patient 1 was diagnosed with stage IIIC1 cancer with grade 2 endometrioid adenocarcinoma. Patient 2 was diagnosed with stage IB undifferentiated endometrial sarcoma. For the ovarian cancer patient, debulking surgery was performed, involving TH, BSO, BPLND, BPALND, omentectomy, multiple biopsy, and washing cytology. Patient 3 was diagnosed with a stage IC bilateral serous borderline tumor. A right salpingo-oophorectomy (RSO) with left ovarian cystectomy was undertaken, and normal-appearing left ovarian tissue was excised. Patient 4 was diagnosed with stage IV right mucinous adenocarcinoma. Grossly normal-appearing right ovarian tissue was excised. Patient 5 was diagnosed with stage IC bilateral clear cell carcinoma. Normal-appearing ovarian tissue was excised bilaterally. Patient 6 was diagnosed with stage IIC double primary left ovarian and stage IIB endometrial endometrioid adenocarcinoma. Cisplatin-based adjuvant chemotherapy was planned after debulking surgery in patients 4,5 , and 6 .

\section{Retrieval of immature oocytes from ovarian tissue}

During the surgical procedure, macroscopically normal ovarian tissue was excised and immediately transferred to the laboratory, with an ischemic time of less than 15 minutes. The macroscopically normal ovarian tissue was harvested, put in a stainless steel bowl filled with $0.9 \% \mathrm{NaCl}$, and was delivered to the laboratory. The removed ovarian tissue was placed in a large petri dish containing warm Quinn's Advantage Medium with HEPES supplemented with heparin. All visible follicles were aspirated with a 10-mL syringe and 18-gauge needle. After ovarian tissue dissection, aspiration was used to randomly to retrieve oocytes beneath the ovarian surface. The aspirate was flushed in Oocyte Washing Medium (CooperSurgical, SAGE, Trumbull, CT, USA) and cumulus-oocyte complexes (COCs) were identified under a dissecting microscope. After oocyte aspiration, the ovarian tissue was also sent for routine pathological assessment to rule out malignant cell infiltration of the primary cancer. In this study, none of the harvested ovarian tissue after oocyte retrieval showed histologic signs of malignant cell infiltration.

\section{Oocyte IVM}

The collected COCs were assessed for the presence or absence of a germinal vesicle or the first polar body by microscopy. The retrieved immature oocytes were isolated and incubated in a four-well dish containing IVM media ( $800 \mu \mathrm{L}$ G2, VitroLife, Kungsbacka, Sweden) supplemented with 10\% Human Serum Albumin (VitroLife), recom- 
binant follicle-stimulating hormone $(75 \mathrm{mlU} / \mathrm{mL}$, Gonal-f, Serono, Rome, Italy), recombinant human chorionic gonadotropin (hCG) (0.5 $\mathrm{IU} / \mathrm{mL}$, Ovidrel, Serono), and estradiol (Sigma, St. Louis, MO, USA) at $37^{\circ} \mathrm{C}$ and $5 \% \mathrm{CO}_{2}$ in humidified air. The mature oocytes were cultured for 4 hours and immature oocytes were cultured for up to 48 hours. After culture, the oocytes were denuded using hyaluronidase solution ( $0.1 \%$ hyaluronidase, Sigma), and their maturity was determined using microscopy by the presence of a first polar body.

\section{Vitrification of IVM oocytes}

The mature oocytes in metaphase 2 were cryopreserved by vitrification. The remaining immature oocytes were cultured for an additional 24 hours, and if any oocytes matured, they were vitrified in the same manner. IVM oocytes were vitrified with a pull-and-cut straw, which was constructed by pulling and cutting a $0.25 \mathrm{~mL}$ plastic sterile straw (Bicef). The oocytes were equilibrated in $7.5 \%$ ethylene glycol (EG) and 7.5\% dimethyl sulfoxide (DMSO) for 20 to 25 minutes, and then exposed to $15 \% \mathrm{EG}$ and $15 \%$ DMSO in phosphate buffered saline (PBS) with $0.5 \mathrm{M}$ sucrose within 1 minute. Then, the vitrified oocytes were immediately plunged into liquid nitrogen. For thawing, oocytes were warmed in $1 \mathrm{M}$ sucrose for 1 minute and in $0.5 \mathrm{M}$ sucrose for 3 minutes, followed by washing in PBS.

\section{Results}

\section{Gynecologic cancer patients}

The characteristics of the patients are presented in Table 1. Oocytes were collected from macroscopically normal ovarian tissue in endometrial $(n=2)$, ovarian $(n=3)$, and double primary ovarian and endometrial $(n=1)$ cancer patients. The patient age at the time of FP consultation ranged from 19 to 39 years, with a mean of 29.0 years.

Patient 1 was a 31-year-old stage IIIC1 endometrial cancer patient.
She was unmarried and had regular menstrual cycles. TH, BSO, BPLND, and BPALND were performed. Immature oocytes were collected on day 7 of her menstrual cycle (MCD 7). Fifteen IVM oocytes out of a total of 19 oocytes were frozen for FP (Fig. 1C). Patient 2 was 29-year-old patient with stage IB endometrial cancer. She had regular menstrual cycles and no coital history. She underwent radical surgery on MCD 3 due to an undifferentiated cell grade. A total of nine oocytes were collected, and five IVM oocytes were frozen. Patient 3 was 19 years old with a stage IC bilateral borderline ovarian tumor. She was unmarried and had 40-day menstrual cycles. Laparoscopic right salpingo-oophorectomy with left ovarian cystectomy was performed in the follicular phase, as suggested by estradiol and progesterone levels of $87.6 \mathrm{pg} / \mathrm{mL}$ and $0.23 \mathrm{ng} / \mathrm{mL}$, respectively. A total of 11 oocytes were collected and seven IVM oocytes were frozen. Patient 4 was 19 years old, with stage IV ovarian cancer. She had no coital history and regular menstrual cycles. Radical surgery was performed on MCD 7. One IVM oocyte out of three immature oocytes was frozen for FP. She underwent six cycles of chemotherapy. Patient 5 was diagnosed with stage IC bilateral clear cell ovarian carcinoma. She was 39 years old and unmarried. Her anti-Müllerian hormone (AMH) levels were less than $0.1 \mathrm{ng} / \mathrm{mL}$. Radical surgery was performed on MCD 9. Oocyte collection was attempted, but failed. Patient 6 was 37 years old, and was diagnosed with stage II double primary ovarian and stage IC endometrial cancer. She was married and had irregular menstrual cycles. A total of 11 oocytes were collected; eight underwent IVM, and five embryos were frozen after fertilization via intracytoplasmic sperm injection (ICSI).

\section{Ex vivo oocyte retrieval and IVM}

All surgical treatments, including oocyte collection, were undertaken during the follicular phase of menstruation. A total of 53 oocytes were retrieved from five patients, with a mean of 10.6 oocytes per

Table 1. Characteristics of the gynecologic cancer patients included in this study

\begin{tabular}{|c|c|c|c|c|c|c|}
\hline Patient no. & Age (yr) & Menstrual cycle (day) & Cancer type & Stage & Operative procedure & Histologic findings \\
\hline 1 & 31 & Regular & Endometrial cancer & IIIC1 & TAH, BSO, BPLND, BPALND & Endometrioid adenocarcinoma (G2) \\
\hline 2 & 29 & Regular & Endometrial cancer & IB & TAH, BSO, BPLND, BPALND, omentectomy & Undifferentiated endometrial sarcoma \\
\hline 3 & 19 & Irregular (40) & Ovarian cancer & IC & $\begin{array}{l}\text { L/S RSO, left ovary cystectomy, } \\
\text { omentectomy, appendectomy }\end{array}$ & Bilateral serous borderline tumor \\
\hline 4 & 19 & Regular & Ovarian cancer & IV & $\begin{array}{l}\text { TAH, BSO, BPLND, BPALND, omentectomy, } \\
\text { appendectomy+CT }\end{array}$ & Right mucinous adenocarcinoma (G3) \\
\hline 5 & 39 & Regular & Ovarian cancer & IC & $\begin{array}{l}\text { LAVH, BSO, BPLND, BPALND, } \\
\text { omentectomy + CT }\end{array}$ & Bilateral clear cell carcinoma \\
\hline 6 & 37 & Irregular (120) & $\begin{array}{l}\text { Double primary tumor } \\
\text { (endometrial and } \\
\text { ovarian cancer) }\end{array}$ & $\| / I C$ & $\begin{array}{l}\text { TAH, BSO, BPLND, BPALND, omentecto- } \\
\text { my, segmental resection of sigmoid } \\
\text { colon+CT }\end{array}$ & $\begin{array}{l}\text { Left endometrioid adenocarcinoma } \\
\text { (G1) }\end{array}$ \\
\hline
\end{tabular}

TAH, total abdominal hysterectomy; BSO, bilateral salpingo-oophorectomy; BPLND, bilateral pelvic lymph node dissection; BPALND, bilateral para-aortic lymph node dissection; G2, grade 2; L/S, laparoscopy; RSO, right salpingo-oophorectomy; CT, chemotherapy; G3, grade 3; LAVH, laparoscopy-assisted vaginal hysterectomy; G1, grade1. 
Table 2. Fertility preservation (FP) options in the cancer patients included in this study

\begin{tabular}{|c|c|c|c|c|c|c|c|c|c|c|}
\hline Patient no. & Age (yr) & Marital status & FP option & $\begin{array}{c}\mathrm{AMH} \\
(\mathrm{ng} / \mathrm{mL})\end{array}$ & $\begin{array}{c}E 2 \\
(p g / m L)\end{array}$ & $\begin{array}{c}\mathrm{P} 4 \\
\text { (ng/mL) }\end{array}$ & MCD & $\begin{array}{l}\text { No. of retrieved } \\
\text { oocytes }\end{array}$ & $\begin{array}{l}\text { No. of IVM } \\
\text { oocytes }\end{array}$ & $\begin{array}{l}\text { Frozen oocytes or } \\
\text { embryos (n) }\end{array}$ \\
\hline 1 & 31 & $S$ & Oocyte freezing & - & - & - & 7 & 19 & 15 & Oocytes (15) \\
\hline 2 & 29 & $S$ & Oocyte freezing & - & - & - & 3 & 9 & 5 & Oocytes (5) \\
\hline 3 & 19 & $S$ & Oocyte freezing & 9.4 & 87.6 & 0.23 & a) & 11 & 7 & Oocytes (7) \\
\hline 4 & 19 & $S$ & Oocyte freezing & - & - & - & 7 & 3 & 1 & Oocytes (1) \\
\hline 5 & 39 & $S$ & Oocyte freezing & $<0.1$ & 583.5 & 0.24 & 9 & Failed & - & - \\
\hline 6 & 37 & $M$ & Embryo freezing & 0.4 & 37 & 0.03 & a) & 11 & 8 & Embryos (5) \\
\hline
\end{tabular}

$\mathrm{AMH}$, anti-Müllerian hormone; $\mathrm{E} 2$, estradiol; $\mathrm{P}$, progesterone; $\mathrm{MCD}$, menstrual cycle day; IVM, in vitro matured; $\mathrm{S}$, single (unmarried); $\mathrm{M}$, married.

a)Follicular phase.
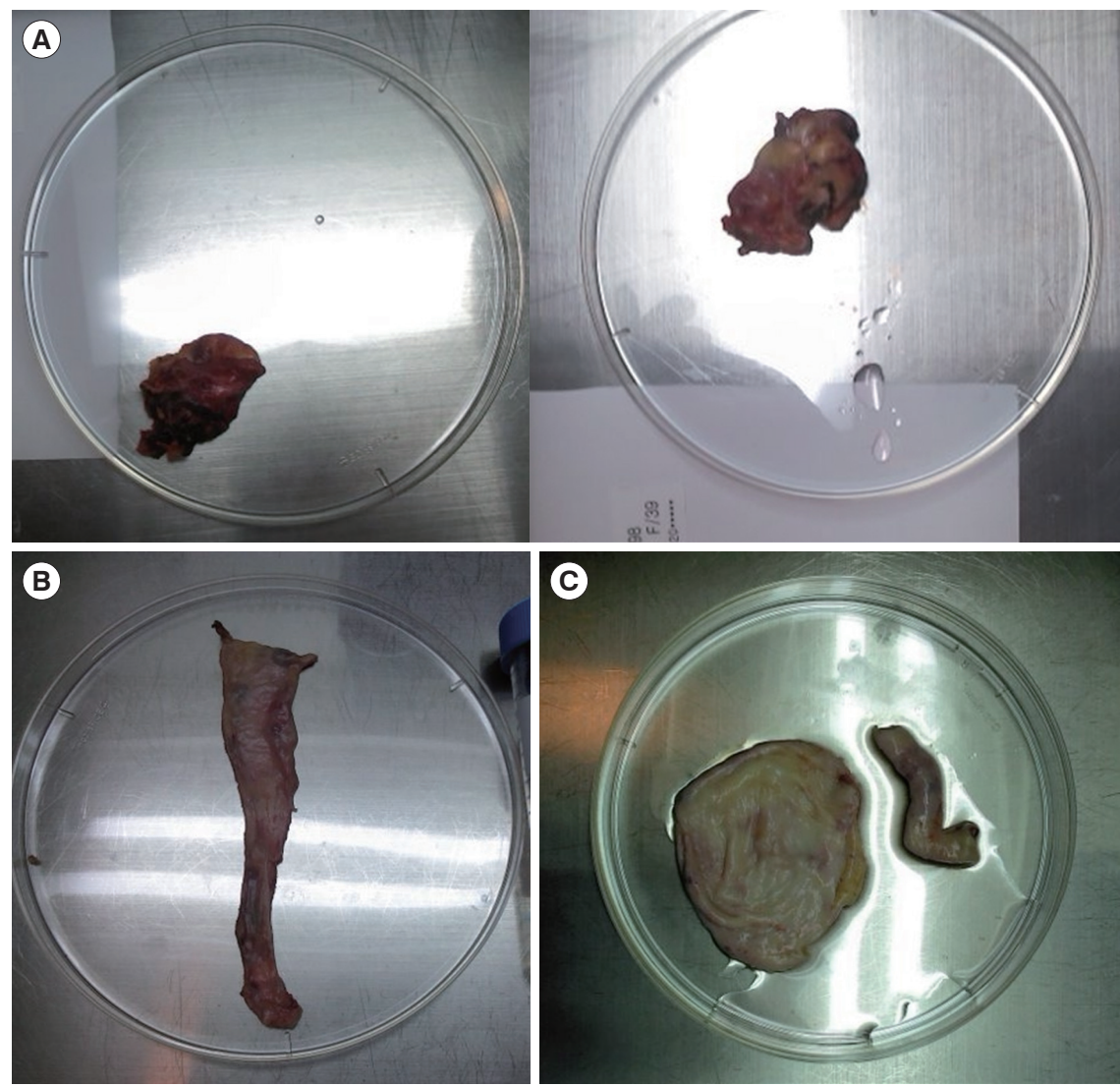

Figure 1. Ovarian tissue harvested during radical surgery. (A) Patient 5 (with ovarian cancer), for whom ex vivo oocyte retrieval failed. (B) Patient 4 (with ovarian cancer), with three immature oocytes retrieved. (C) Patient 1 (with endometrial cancer), with 19 immature oocytes retrieved.

patient. After IVM, a total of 36 mature oocytes were obtained, demonstrating a $67.9 \%$ maturation rate. One to 15 IVM oocytes were frozen per patient (Table 2).

For patient 6, five embryos were frozen after ICSI was performed in eight IVM oocytes. For patient 5, with stage IC ovarian cancer, the harvested ovarian tissue volume was small and fibrotic, which may explain the failure to collect oocytes from this sample (Figure 1A). More oocytes were frozen from patient 3 , whose harvested ovarian tissue volume was larger than that of patient 5 , although these pa- tients had the same stage of cancer. One matured oocyte was frozen from patient 4, who had stage IV cancer, whereas a total of seven oocytes were frozen from Patient 3, whose cancer was stage IC, despite the fact that these patients were of a similar age (Figure 1B).

\section{Discussion}

Significant progress has taken place in the area of assisted reproductive technology towards FP, which helps female cancer patients 
maintain fertility in the face of cancer treatment. This study evaluated the feasibility of oocyte IVM and embryo freezing in gynecologic cancer patients undergoing radical surgery. Immature oocytes were successfully retrieved from macroscopically normal harvested ovarian tissue. After harvesting and IVM, the oocytes were frozen, and embryo freezing was possible with ICSI using IVM oocytes for the one patient who was married.

Gynecologic cancer treatment has traditionally been focused only on eradicating disease, with surgical treatment as a crucial component in the treatment. Uterine or ovarian cancer is staged surgically and the pathologic assessment of organs in situ is necessary to determine the prognosis and the proper adjuvant therapy. In certain early stage cancers, therapeutic surgical procedures can be modified in order to preserve fertility; however, in the case of advanced-stage cancers, radical surgery remains mandatory, and the reproductive organs must be removed. Radical surgery not only eliminates the possibility of future fertility, but is also associated with significant surgical morbidity.

Advanced-stage cancer patients are required to undergo surgical treatment as soon as possible. It is often difficult for oncologists to bring up the topic of FP because the diagnosis of advanced-stage cancer can itself be overwhelming for the patient. Patients are not likely to be counseled regarding FP and have little opportunity to express concerns about their quality of life after cancer treatment. However, if the opportunity is available, oocyte harvesting is possible before or at the time of radical surgery, and embryo freezing can be an option for FP, in order to allow future pregnancies to occur with a surrogate mother.

We believe that oocyte freezing should at least be proposed to gynecologic cancer patients. Mature oocyte freezing after controlled ovarian stimulation (COS) is the most preferable method for oocyte freezing [12]. However, in the case of radical cancer patients with time constraints for $\mathrm{FP}, \mathrm{COS}$, which requires seven to 10 days, is not possible; moreover, the high estradiol levels associated with $\mathrm{COS}$ are deemed unsafe for patients with hormone-sensitive tumors. Immature oocyte collection without COS, followed by IVM oocyte freezing, is an alternative option. This technique has been used in conjunction with ovarian tissue freezing. IVM has been offered as an additional strategy to increase the efficacy of freezing the ovarian tissue [8]. As the antral follicles are devoid of cancer cell infiltration, oocytes retrieved from the antral follicles offer a reliable option in the case of cancer patients with a high potential for ovarian involvement or in situ ovarian cancer.

Oocytes can be collected in vivo using transvaginal ultrasoundguided aspiration. In the case of ovarian malignancies, in vivo oocyte collection may result in the spillage of cancer cells. The possible occurrence of malignant cancer cell spillage is of concern, since it may result in intra-peritoneal dissemination of the cancer. According to the Fédération Internationale de Gynécologie et d'Obstétrique classification, rupture of a malignant tumor apparently confined to one ovary during surgery advances the disease to stage IC. In the case of clear cell carcinomas of the ovary, capsule status is an independent prognostic factor predictive of poor overall and disease-free survival [13]. Therefore, ex vivo oocyte retrieval is considered the safest option for avoiding malignant cell spillage into the peritoneal cavity.

Immature oocytes were successfully collected from macroscopically normal ovarian tissue in five out of six patients, with a mean of 10.6 oocytes. No oocytes were retrieved from one patient with an $\mathrm{AMH}$ level $<0.1 \mathrm{ng} / \mathrm{mL}$ and an ovarian reserve that was compromised in comparison with an age-matched woman. Although it is not clear whether the failed oocyte collection was due to decreased ovarian reserve or the small fibrotic ovarian tissue, recent reports have suggested that ovarian reserve is diminished in cancer patients [14-16]. The lower ovarian reserve in cancer patients may be explained by either accelerated follicle loss or a defect in the recruitment of antral follicles due to the disease. In a recent meta-analysis of seven retrospective studies, female cancer patients had lower numbers of total oocytes and mature oocytes after COS for FP than healthy agematched patients, reflecting decreased ovarian reserve [17].

In ovarian cancer patients, the number of retrieved oocytes may be negatively associated with cancer stage. We observed a trend for a higher cancer stage to be associated with a lower number of retrieved oocytes. Eleven oocytes were retrieved from patient 3, whose cancer was stage IC, while three oocytes were retrieved from patient 4, whose cancer was stage IV, despite being approximately the same age. The volume of harvested ovarian tissue is another factor that determines the possibility of oocyte collection. In the case of a cancer patient whose ovarian cortical surface was smooth and devoid of antral follicles, harvesting resulted in the collection of only three oocytes, from which only one IVM oocyte was frozen, although the patient was 19 years of age. Within ovaries, the secondary and antral follicles contain immature oocytes, which account for approximately $7 \%$ of the follicular population. Therefore, the tissue volume reflects the follicular population $[18,19]$.

FP offers the hope of future fertility to cancer survivors. At an appropriate time after cancer treatment, frozen oocytes can be thawed, fertilized, and implanted in surrogate mothers, with the hope that this process will result in a live birth. The success rates for live births using frozen IVM oocytes are improving, but still remain low, at approximately $20 \%[20,21]$. Maximizing the number of oocytes for freezing is therefore extremely important, because most patients have a single chance for FP due to time constraints. Surplus oocyte collection is the first step for successful FP. COS with gonadotropins is a major strategy used to increase the oocyte collection per cycle, 
meaning that IVM without COS has limitations in terms of the oocyte quantity. For this reason, earlier referrals should be considered for $\mathrm{COS}$ in order to maximize the number of oocytes retrieved [22]. Although it is ideal to start $\operatorname{COS}$ within 3 days of menstruation in order to retrieve multiple oocytes, the recent introduction of random-start stimulation has overcome the restriction of the starting cycle day. $\mathrm{COS}$ can be started in the follicular or luteal phase regardless of the menstrual cycle [23-26]. Another approach involves oocyte retrieval with hCG priming 36 to 38 hours prior to collection in order to increase the maturity of the oocytes $[27,28]$. In this study, none of the patients had 36 hours available to wait from hCG injection to retrieval.

Retrieved immature oocytes can be frozen either at the immature germinal vesicle stage or at the mature metaphase II stage after IVM. It has been proposed that immature oocyte freezing, instead of IVM oocyte freezing, may reduce the risk of aneuploidy [29]. However, the literature to date indicates that the potential for oocyte maturation after thawing frozen immature oocytes is significantly reduced [30]. Therefore, IVM oocyte freezing is favored over immature oocyte freezing.

Although an oocyte maturation rate of $67.9 \%$ is encouraging, ex vivo IVM oocyte quality can be a major concern in comparison with in vivo IVM oocytes. The risk of meiotic disturbances due to temperature changes experienced during the transition from in vivo to ex vivo cannot be neglected [8].

Ex vivo oocyte retrieval followed by IVM oocyte freezing is a feasible option for FP. This option is best offered to gynecologic cancer patients with time constraints, cancers with a high potential of ovarian involvement, or in situ ovarian cancer.

\section{Conflict of interest}

No potential conflict of interest relevant to this article was reported.

\section{References}

1. American Cancer Society. Cancer facts and figures 2010 [Internet]. Atlanta: American Cancer Society; 2010 [cited Sep 2014]. Available from: http://www.cancer.org/ acs/groups/content/@ nho/documents/document/acspc-024113.pdf.

2. Makar AP, Trope C. Fertility preservation in gynecologic cancer. Acta Obstet Gynecol Scand 2001;80:794-802.

3. Gershenson DM. Fertility-sparing surgery for malignancies in women. J Natl Cancer Inst Monogr 2005;(34):43-7.

4. Rasool N, Rose PG. Fertility-preserving surgical procedures for patients with gynecologic malignancies. Clin Obstet Gynecol 2010:53:804-14.
5. Noyes N, Knopman JM, Long K, Coletta JM, Abu-Rustum NR. Fertility considerations in the management of gynecologic malignancies. Gynecol Oncol 2011;120:326-33.

6. National Institutes of Health. SEER cancer statistics [Internet]. Bethesda: National Institutes of Health; [cited Sep 2014]. Available from: $h$ ttp://seer.cancer.gov .

7. Marhhom E, Cohen I. Fertility preservation options for women with malignancies. Obstet Gynecol Surv 2007;62:58-72.

8. Huang JY, Tulandi T, Holzer H, Tan SL, Chian RC. Combining ovarian tissue cryobanking with retrieval of immature oocytes followed by in vitro maturation and vitrification: an additional strategy of fertility preservation. Fertil Steril 2008;89:567-72.

9. Revel A, Safran A, Benshushan A, Shushan A, Laufer N, Simon A. In vitro maturation and fertilization of oocytes from an intact ovary of a surgically treated patient with endometrial carcinoma: case report. Hum Reprod 2004;19:1608-11.

10. Fadini R, Dal Canto M, Mignini Renzini M, Milani R, Fruscio R, Cantu MG, et al. Embryo transfer following in vitro maturation and cryopreservation of oocytes recovered from antral follicles during conservative surgery for ovarian cancer. J Assist Reprod Genet 2012;29:779-81.

11. Prasath EB, Chan ML, Wong WH, Lim CJ, Tharmalingam MD, Hendricks $M$, et al. First pregnancy and live birth resulting from cryopreserved embryos obtained from in vitro matured oocytes after oophorectomy in an ovarian cancer patient. Hum Reprod 2014;29:276-8.

12. Practice Committees of American Society for Reproductive Medicine; Society for Assisted Reproductive Technology. Mature oocyte cryopreservation: a guideline. Fertil Steril 2013;99:37-43.

13. Higashi M, Kajiyama H, Shibata K, Mizuno M, Mizuno K, Hosono S, et al. Survival impact of capsule rupture in stage I clear cell carcinoma of the ovary in comparison with other histological types. Gynecol Oncol 2011;123:474-8.

14. Lawrenz B, Fehm T, von Wolff M, Soekler M, Huebner S, Henes J, et al. Reduced pretreatment ovarian reserve in premenopausal female patients with Hodgkin lymphoma or non-Hodgkin-lymphoma: evaluation by using antimullerian hormone and retrieved oocytes. Fertil Steril 2012;98:141-4.

15. Ebbel E, Katz A, Kao CN, Cedars M. Reproductive aged women with cancer have a lower antral follicle count than expected. Fertil Steril 2011;96:S199-200.

16. Frattarelli JL, Levi AJ, Miller BT, Segars JH. A prospective assessment of the predictive value of basal antral follicles in in vitro fertilization cycles. Fertil Steril 2003;80:350-5.

17. Friedler S, Koc O, Gidoni Y, Raziel A, Ron-El R. Ovarian response to stimulation for fertility preservation in women with malignant disease: a systematic review and meta-analysis. Fertil Steril 2012; 
97:125-33.

18. Gougeon A. Dynamics of follicular growth in the human: a model from preliminary results. Hum Reprod 1986;1:81-7.

19. Lass A, Silye R, Abrams DC, Krausz T, Hovatta O, Margara R, et al. Follicular density in ovarian biopsy of infertile women: a novel method to assess ovarian reserve. Hum Reprod 1997;12:102831.

20. Chian RC, Gilbert L, Huang JY, Demirtas E, Holzer H, Benjamin A, et al. Live birth after vitrification of in vitro matured human oocytes. Fertil Steril 2009;91:372-6.

21. Chian RC, Huang JY, Gilbert L, Son WY, Holzer H, Cui SJ, et al. Obstetric outcomes following vitrification of in vitro and in vivo matured oocytes. Fertil Steril 2009;91:2391-8.

22. Lee S, Ozkavukcu S, Heytens E, Moy F, Oktay K. Value of early referral to fertility preservation in young women with breast cancer. J Clin Oncol 2010;28:4683-6.

23. von Wolff M, Thaler CJ, Frambach T, Zeeb C, Lawrenz B, Popovici RM, et al. Ovarian stimulation to cryopreserve fertilized oocytes in cancer patients can be started in the luteal phase. Fertil Steril 2009;92:1360-5.

24. Bedoschi GM, de Albuquerque FO, Ferriani RA, Navarro PA. Ovarian stimulation during the luteal phase for fertility preservation of cancer patients: case reports and review of the literature. J As- sist Reprod Genet 2010;27:491-4.

25. Sonmezer M, Turkcuoglu I, Coskun U, Oktay K. Random-start controlled ovarian hyperstimulation for emergency fertility preservation in letrozole cycles. Fertil Steril 2011;95:2125.e9-11.

26. Nayak SR, Wakim AN. Random-start gonadotropin-releasing hormone (GnRH) antagonist-treated cycles with $\mathrm{GnRH}$ agonist trigger for fertility preservation. Fertil Steril 2011;96:e51-4.

27. Huang JY, Chian RC, Gilbert L, Fleiszer D, Holzer H, Dermitas E, et al. Retrieval of immature oocytes from unstimulated ovaries followed by in vitro maturation and vitrification: a novel strategy of fertility preservation for breast cancer patients. Am J Surg 2010; 200:177-83.

28. Shalom-Paz E, Almog B, Shehata F, Huang J, Holzer H, Chian RC, et al. Fertility preservation for breast-cancer patients using IVM followed by oocyte or embryo vitrification. Reprod Biomed Online 2010;21:566-71.

29. Mandelbaum J, Anastasiou O, Levy R, Guerin JF, de Larouziere V, Antoine JM. Effects of cryopreservation on the meiotic spindle of human oocytes. Eur J Obstet Gynecol Reprod Biol 2004;113 Suppl 1:S17-23.

30. Fasano G, Demeestere I, Englert Y. In-vitro maturation of human oocytes: before or after vitrification? J Assist Reprod Genet 2012; 29:507-12. 